\title{
EFFECTS OF PIEZOELECTRICITY ON BULK WAVES IN MONOCLINIC PORO-ELASTIC MATERIALS
}

\author{
VISHAKHA GUPTA \\ Dyal Singh College, Department of Mathematics, Karnal, India \\ AnIL K. VAShishth \\ Kurukshetra University, Department of Mathematics, Kurukshetra, India \\ e-mail:vi_shu85@yahoo.co.in
}

\begin{abstract}
Piezoelectric materials are materials which produce electric field when stress is applied and get strained when electric field is applied. Piezoelectric materials are acting as very important functional components in sonar projectors, fluid monitors, pulse generators and surface acoustic wave devices. Wave propagation in porous piezoelectric material having crystal symmetry 2 is studied analytically. The Christoffel equation is derived. The phase velocities of propagation of all these waves are described in terms of complex wave velocities. The effects of phase direction, porosity, wave frequency and piezoelectric interaction on the phase velocities are studied numerically for a particular model.
\end{abstract}

Keywords: piezoelectricity, porous, monoclinic

\section{Introduction}

Piezoelectric materials have widespread applications in many branches of science and technology such as electronics, navigation, mechatronics and micro-system technology. In recent years, piezoelectric materials have been integrated with structural systems to form a class of smart structures and embedded as layers or fibers into multifunctional composites. The survey of literature can be found in many related texts and books (Arnau, 2008; Auld, 1973). A short survey of the piezoelectric wave propagation and resonance were described by Auld (1981). Both, theoretical and experimental studies on wave propagation in piezoelectric materials have attracted attention of scientists and engineers during the last two decades. Nayfeh and Chien (1992) made a study on ultrasonic wave interaction with fluid-loaded anisotropic piezoelectric substrates and derived an analytical expression for reflection and transmission coefficients for monoclinic materials. Zinchuk and Podlipenents (2001) obtained dispersion equations for the acousto-electric Rayleigh wave in a periodic layer piezoelectric half-space in a study for a $6 \mathrm{~mm}$ crystal class.

Porous piezoelectric materials (PPM) are widely used for applications such as low frequency hydrophones, miniature, accelerometers, vibratory sensors and contact microphones. Experimental studies (Qian et al., 2004; Praveenkumar et al., 2005; Piazza et al., 2006) related to properties of porous piezoelectric materials and influence of porosity on its properties have been made by different authors. Gupta and Venkatesh (2006) developed a finite element model to study the effect of porosity on the electromechanical response of piezoelectric materials. Craciun et al. (1998) and Gomez et al. (2000) made an experimental study on wave propagation in porous piezoceramics. Vashishth and Gupta (2009a) derived constitutive equations for anisotropic porous piezoelectric materials. Wave propagation in transversely isotropic porous piezoelectric materials was studied analytically and numerically by Vashishth and Gupta (2009b).

In this paper, the wave propagation in porous piezoelectric materials having crystal symmetry 2 is studied. The constitutive equations are formulated for porous piezoelectric materials 
having crystal symmetry 2. The Christoffel equation is derived analytically and its solutions are obtained numerically. The variation of phase velocities with the direction of propagation, porosity, piezoelectricity and viscosity is studied numerically for a particular model.

\section{Governing equations and their solution}

The constitutive equations for anisotropic porous piezoelectric materials (Vashishth and Gupta, 2009a) are

$$
\begin{array}{rlrl}
\sigma_{i j} & =c_{i j k l} \varepsilon_{k l}+m_{i j} \varepsilon^{*}+e_{k i j} \phi_{, k}+\zeta_{k i j} \phi_{, k}^{*} & & \sigma^{*}=m_{i j} \varepsilon_{i j}+R \varepsilon^{*}+\widetilde{\zeta}_{i} \phi_{, i}+e_{i}^{*} \phi_{, i}^{*} \\
D_{i}=e_{i j k} \varepsilon_{j k}+\widetilde{\zeta}_{i} \varepsilon^{*}-\xi_{i j} \phi_{, j}-A_{i j} \phi_{, j}^{*} & D_{i}^{*}=\zeta_{i j k} \varepsilon_{j k}+e_{i}^{*} \varepsilon^{*}-A_{i j} \phi_{, j}-\xi_{i j}^{*} \phi_{, j}^{*}
\end{array}
$$

where $\sigma_{i j} / \sigma^{*}$ are the stress components acting on the solid/fluid phase of a porous aggregate. $\varepsilon_{i j} / \varepsilon^{*}$ are strain tensor components for the solid/fluid phase, respectively. $\phi / \phi^{*}$ and $D_{i} / D_{i}^{*}$ are electric potentials and electric displacement components for the solid/fluid phase of the porous bulk material, respectively. $c_{i j k l}$ are elastic stiffness constants. The elastic constant $R$ measures the pressure to be exerted on the fluid to push its unit volume into the porous matrix. $e_{k i j} / e_{i}^{*}, \xi_{i j} / \xi_{i j}^{*}$ are piezoelectric and dielectric constants for the solid/fluid phase, respectively. $m_{i j} ; \zeta_{k i j}, \widetilde{\zeta}_{i} ; A_{i j}$ are the parameters which take into account the elastic; piezoelectric; dielectric coupling between the two phases of the porous aggregate.

The coefficient matrix for porous piezoelectric materials, having crystal symmetry 2 (Auld, 1973) is

$$
\left[\begin{array}{ccccccccccccc}
c_{11} & c_{12} & c_{13} & 0 & 0 & c_{16} & m_{11} & 0 & 0 & -e_{31} & 0 & 0 & -\zeta_{31} \\
c_{12} & c_{22} & c_{23} & 0 & 0 & c_{26} & m_{11} & 0 & 0 & -e_{32} & 0 & 0 & -\zeta_{32} \\
c_{13} & c_{23} & c_{33} & 0 & 0 & c_{36} & m_{33} & 0 & 0 & -e_{33} & 0 & 0 & -\zeta_{33} \\
0 & 0 & 0 & c_{44} & c_{45} & 0 & 0 & -e_{14} & -e_{24} & 0 & -\zeta_{14} & -\zeta_{24} & 0 \\
0 & 0 & 0 & c_{45} & c_{55} & 0 & 0 & -e_{15} & -e_{25} & 0 & -\zeta_{15} & -\zeta_{25} & 0 \\
0 & 0 & 0 & 0 & 0 & c_{66} & m_{12} & 0 & 0 & -e_{36} & 0 & 0 & -\zeta_{36} \\
m_{11} & m_{11} & m_{33} & 0 & 0 & m_{12} & R & 0 & 0 & -\widetilde{\zeta}_{3} & 0 & 0 & -e_{3}^{*} \\
0 & 0 & 0 & e_{14} & e_{15} & 0 & 0 & \xi_{11} & \xi_{12} & 0 & A_{11} & A_{12} & 0 \\
0 & 0 & 0 & e_{24} & e_{25} & 0 & 0 & \xi_{12} & \xi_{22} & 0 & A_{12} & A_{22} & 0 \\
e_{31} & e_{32} & e_{33} & 0 & 0 & e_{36} & \widetilde{\zeta}_{3} & 0 & 0 & \xi_{33} & 0 & 0 & A_{33} \\
0 & 0 & 0 & \zeta_{14} & \zeta_{15} & 0 & 0 & A_{11} & A_{12} & 0 & \xi_{11}^{*} & \xi_{12}^{*} & 0 \\
0 & 0 & 0 & \zeta_{24} & \zeta_{25} & 0 & 0 & A_{12} & A_{22} & 0 & \xi_{12}^{*} & \xi_{22}^{*} & 0 \\
\zeta_{31} & \zeta_{32} & \zeta_{33} & 0 & 0 & 0 & e_{3}^{*} & 0 & 0 & A_{33} & 0 & 0 & \xi_{33}^{*}
\end{array}\right]
$$

The equations of motion for a fluid-saturated porous piezoelectric medium, in the absence of body forces, are (Vashishth and Gupta, 2009a)

$$
\begin{aligned}
\sigma_{i j, j} & =\rho_{11} \ddot{u}_{j}+\rho_{12} \ddot{U}_{j}^{*} & & \sigma_{, i}^{*}=\rho_{12} \ddot{u}_{j}+\rho_{22} \ddot{U}_{j}^{*} \\
D_{i, i} & =0 & & D_{i, i}^{*}=0
\end{aligned}
$$

where $u_{i} / U_{i}^{*}$ are the components of mechanical displacement for the solid/fluid phase of the porous aggregate. $\rho_{11}, \rho_{12}$ and $\rho_{22}$ are dynamical coefficients.

For the propagation of plane waves, let us assume that

$$
\begin{array}{ll}
u_{j}=B_{j} \exp \left[\mathrm{i} \omega\left(p_{k} x_{k}-t\right)\right] & U_{j}^{*}=F_{j} \exp \left[\mathrm{i} \omega\left(p_{k} x_{k}-t\right)\right] \\
\phi=G \exp \left[\mathrm{i} \omega\left(p_{k} x_{k}-t\right)\right] & \phi^{*}=H \exp \left[\mathrm{i} \omega\left(p_{k} x_{k}-t\right)\right]
\end{array}
$$


where $\mathrm{i}=\sqrt{-1}, p_{k}$ are the components of the slowness vector $\mathbf{p}$. These can be written as $p_{k}=n_{k} / V$ in terms of phase velocity $v$, where $n_{k}$ are components of the unit vector normal to the wave surface. $\omega$ is the circular frequency of waves and $t$ is the time. Making use of equations (2.1)-(2.4), we obtain a system of equations in unknowns $B_{j}, F_{j}(j=1,2,3), G$ and $H$, which is given as follows

$$
\begin{aligned}
& \left(c_{11} n_{1}^{2}+c_{55} n_{3}^{2}-\rho_{11} v^{2}\right) B_{1}+\left(c_{16} n_{1}^{2}+c_{45} n_{3}^{2}\right) B_{2}+\left[\left(c_{13}+c_{55}\right) n_{1} n_{3}\right] B_{3} \\
& \quad+\left(m_{11} n_{1}^{2}-\rho_{12} v^{2}\right) F_{1}+\left(m_{11} n_{1} n_{3}\right) F_{3}+\left[\left(e_{31}+e_{15}\right) n_{1} n_{3}\right] G+\left[\left(\zeta_{31}+\zeta_{15}\right) n_{1} n_{3}\right] H=0 \\
& \left(c_{16} n_{1}^{2}+c_{45} n_{3}^{2}\right) B_{1}+\left(c_{66} n_{1}^{2}+c_{44} n_{3}^{2}-\rho_{11} v^{2}\right) B_{2}+\left[\left(c_{36}+c_{45}\right) n_{1} n_{3}\right] B_{3}+\left(m_{12} n_{1}^{2}\right) F_{1} \\
& \quad+\left(m_{12} n_{1} n_{3}\right) F_{3}+\left[\left(e_{36}+e_{14}\right) n_{1} n_{3}\right] G+\left[\left(\zeta_{36}+\zeta_{14}\right) n_{1} n_{3}\right] H=0 \\
& {\left[\left(c_{13}+c_{55}\right) n_{1} n_{3}\right] B_{1}+\left[\left(c_{36}+c_{45}\right) n_{1} n_{3}\right] B_{2}+\left(c_{55} n_{1}^{2}+c_{33} n_{3}^{2}-\rho_{11} v^{2}\right) B_{3}+\left(m_{33} n_{1} n_{3}\right) F_{1}} \\
& \quad+\left(m_{33} n_{3}^{2}-\rho_{12} v^{2}\right) F_{3}+\left(e_{15} n_{1}^{2}+e_{33} n_{3}^{2}\right) G+\left(z e t a_{15} n_{1}^{2}+\zeta_{33} n_{3}^{2}\right) H=0 \\
& \left(m_{11} n_{1}^{2}-\rho_{12} v^{2}\right) B_{1}+\left(m_{12} n_{1}^{2}\right) B_{2}+\left(m_{33} n_{1} n_{3}\right) B_{3}+\left(R n_{1}^{2}-\rho_{22} v^{2}\right) F_{1} \\
& \quad+\left(R n_{1} n_{2}\right) F_{2}+\left(R n_{1} n_{3}\right) F_{3}+\left(\widetilde{\zeta}_{3} n_{1} n_{3}\right) G+\left(e_{3}^{*} n_{1} n_{3}\right) H=0 \\
& \rho_{12} B_{2}+\rho_{22} F_{2}=0 \\
& \left(m_{11} n_{1} n_{3}\right) B_{1}+\left(m_{12} n_{1} n_{3}\right) B_{2}+\left(m_{33} n_{3}^{2}-\rho_{12} v^{2}\right) B_{3}+\left(R n_{1} n_{3}\right) F_{1} \\
& \quad+\left(R n_{3}^{2}-\rho_{22} v^{2}\right) F_{3}+\left(\widetilde{\zeta}_{3} n_{3}^{2}\right) G+\left(e_{3}^{*} n_{3}^{2}\right) H=0 \\
& \quad\left[\left(e_{15}+e_{31}\right) n_{1} n_{3}\right] B_{1}+\left[\left(e_{14}+e_{36}\right) n_{1} n_{3}\right] B_{2}+\left(e_{15} n_{1}^{2}+e_{33} n_{3}^{2}\right) B_{3}+\left(\widetilde{\zeta}_{3} n_{1} n_{3}\right) F_{1} \\
& \quad+\left(\widetilde{\zeta}_{3} n_{3}^{2}\right) F_{3}-\left(\xi_{11} n_{1}^{2}+\xi_{33} n_{3}^{2}\right) G-\left(A_{11} n_{1}^{2}+A_{33} n_{3}^{2}\right) H=0 \\
& {\left[\left(\zeta_{15}+\zeta_{31}\right) n_{1} n_{3}\right] B_{1}+\left[\left(\zeta_{14}+\zeta_{36}\right) n_{1} n_{3}\right] B_{2}+\left(\zeta_{15} n_{1}^{2}+\zeta_{33} n_{3}^{2}\right) B_{3}+\left(e_{3}^{*} n_{1} n_{3}\right) F_{1}} \\
& \quad+\left(e_{3}^{*} n_{3}^{2}\right) F_{3}-\left(A_{11} n_{1}^{2}+A_{33} n_{3}^{2}\right) G-\left(\xi_{11}^{*} n_{1}^{2}+\xi_{33}^{*} n_{3}^{2}\right) H=0
\end{aligned}
$$

The condition of existence of a non-trivial solution of the system leads to

$$
x_{1} V^{8}+x_{2} V^{6}+x_{3} V^{4}+x_{4} V^{2}+x_{5}=0
$$

where $x_{1}, x_{2}, x_{3}, x_{4}, x_{5}$ are coefficients which have been calculated symbolically.

On solving equation (2.6), we obtain 4 complex roots $V_{j}(j=1,2,3,4)$. Corresponding to these 4 complex roots, we get 4 complex wave velocities $v_{j}$ of four waves. Thus we obtain four plane harmonic waves propagating along the given phase direction in the monoclinic porous piezoelectric material. The wave with the largest phase velocity is termed as stiffened quasi- $P_{1}$ wave, and the wave with the smallest phase velocity is termed as quasi- $P_{2}$ wave. The other two waves are termed as quasi- $S_{1}$ and quasi- $S_{2}$ waves.

\section{Numerical discussion}

The analytical expressions of the phase velocity of propagation and the attenuation quality factor of stiffened quasi $P_{1}, P_{2}$ and $S_{1}, S_{2}$ waves are computed numerically for a particular model Barium Sodium Niobate. Following Auld (1973), the elastic, piezoelectric and dielectric constants for the monoclinic crystal are given in Table 1.

Figure 1 exhibits the variation of phase velocities of quasi waves in porous piezoelectric materials saturated with a viscous fluid for the crystal class 2, respectively with the direction of propagation $(\theta, \phi)$. It is seen from these figures that the range of variation of the velocities of four waves are different. The elevations and depressions of phase velocity surfaces from the horizontal plane in the figures measure the extent of velocity anisotropy in the medium. The effects of azimuth variation on the phase velocities of $q P_{1}$ and $q P_{2}$ waves are negligible for small 
Table 1. Elastic constants, piezoelectric constants and dielectric constants of Barium Sodium Niobate crystal

\begin{tabular}{|c|c|c|}
\hline $\begin{array}{c}\text { Elastic constants } \\
{[\mathrm{GPa}]}\end{array}$ & $\begin{array}{c}\text { Piezoelectric constants } \\
{\left[\mathrm{C} / \mathrm{m}^{2}\right]}\end{array}$ & $\begin{array}{c}\text { Dielectric constants } \\
{[\mathrm{nC} /(\mathrm{Vm})]}\end{array}$ \\
\hline \hline$c_{11}=150.4$ & $e_{15}=11.4$ & $\xi_{11}=10.8$ \\
$c_{12}=60.63$ & $e_{24}=15.4$ & $\xi_{13}=9.8$ \\
$c_{13}=65.94$ & $e_{31}=-4.32$ & $\xi_{22}=14.8$ \\
$c_{22}=160.4$ & $e_{32}=-6.32$ & $\xi_{33}=13.1$ \\
$c_{23}=75.94$ & $e_{33}=17.4$ & $\xi_{11}^{*}=0.038$ \\
$c_{33}=145.5$ & $e_{36}=0.8$ & $\xi_{13}^{*}=0.09$ \\
$c_{44}=40.86$ & $\zeta_{15}=0.456$ & $\xi_{22}^{*}=0.055$ \\
$c_{55}=43.86$ & $\zeta_{24}=0.356$ & $\xi_{33}^{*}=0.049$ \\
$c_{66}=50.37$ & $\zeta_{31}=-1.728$ & $A_{11}=0.018$ \\
$m_{11}=8.8$ & $\zeta_{32}=-0.2728$ & $A_{13}=0.04$ \\
$m_{13}=11.5$ & $\zeta_{33}=0.696$ & $A_{22}=0.031$ \\
$m_{22}=16.8$ & $\zeta_{36}=0.08$ & $A_{33}=0.015$ \\
$m_{33}=5.2$ & $e_{3}^{*}=-3.6$ & \\
$R=20$ & $\widetilde{\zeta}_{3}=-7.5$ & \\
\hline
\end{tabular}

values of $\theta$ but noticeable for large values of $\theta$. The phase velocity of $q P_{1}$ wave an increases with increase in $\theta$ but the phase velocity of $q P_{2}$ wave decreases with an increase in $\theta$. The phase velocity of $q S_{1}$ wave first decreases with an increase in $\theta$ and attain local minima at $\theta=30^{\circ}$ and after that it increases with $\theta$. Contrary to this, $V_{4}$ increases first and then decreases having the minimum at $45^{\circ}$ and then increases further.

(a)

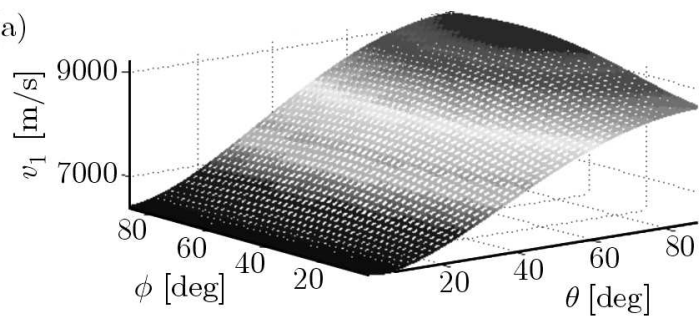

(c)

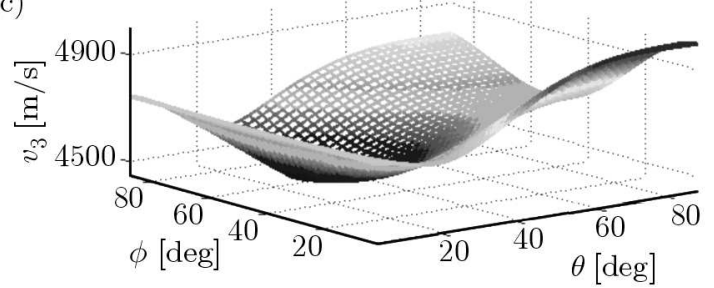

(b)

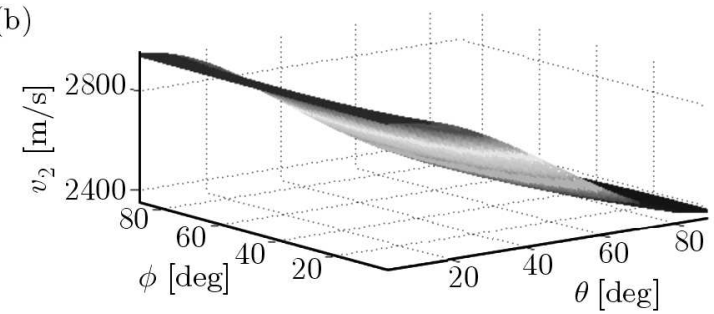

(d)

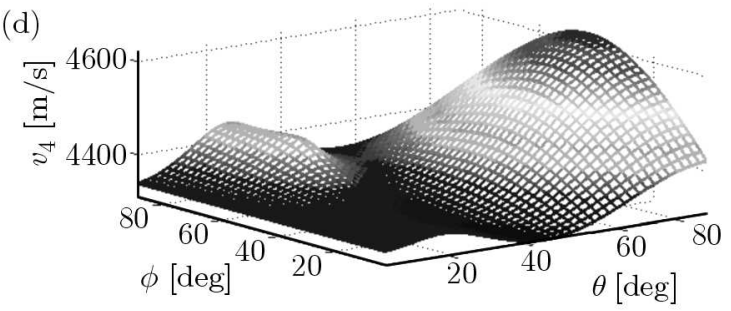

Fig. 1. Variation of phase velocities of quasi waves with the propagation directions $(\theta, \phi)$ for Barium Sodium Niobate crystal of class 2; (a) $q P_{1}$ wave, (b) $q P_{2}$ wave, (c) $q S_{1}$ wave, (d) $q S_{2}$ wave

Figure 2a depicts the variation of phase velocities with the direction of propagation in PPM saturated with a non-viscous fluid, for the crystal class 2 . The waves are not attenuated in such a medium. Figures $2 \mathrm{~b}$ and $2 \mathrm{c}$ exhibit the variation of phase velocities in PPM saturated with the viscous fluid in the low frequency range (LFR) and high frequency range (HFR), respectively. It is observed that all waves slow down due to the viscous effects of the pore fluid. It is also observed that the faster the wave is, the larger are effects of viscosity. It is also interesting to note that the effects of viscosity are not significant in the HFR which reveals the fact that viscosity 
dominates in LFR. Comparison of Figs. 2b and 2c shows that the velocities of all the quasi waves increase as the frequency shifts from LFR to HFR. The pattern of variation of phase velocities with the phase directions remain unaffected in either case.

(a)
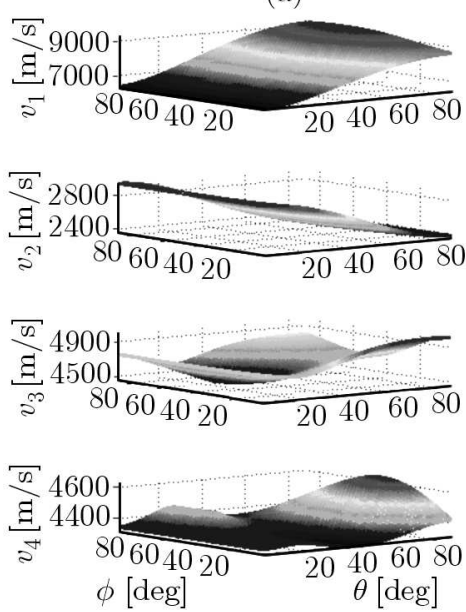

(b)
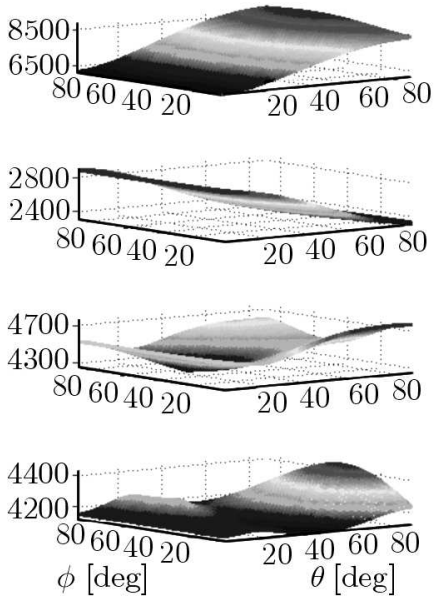

(c)
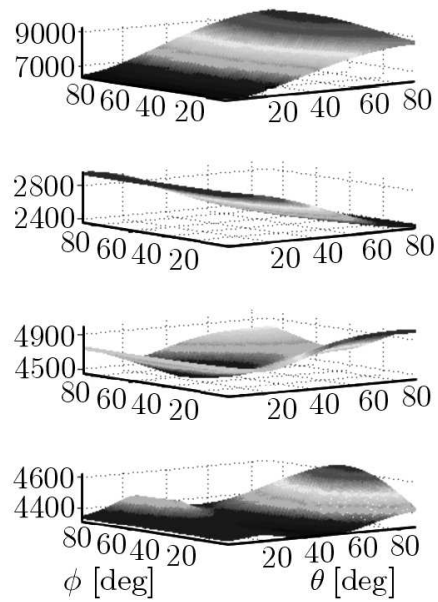

Fig. 2. Effects of viscosity of the pore fluid on the variation of phase velocities of quasi waves with the direction of propagation $(\theta, \phi)$ for Barium Sodium Niobate crystal of class 2; (a) without viscous effects, (b) with viscous effects in the low frequency range, (c) with viscous effects in the high frequency range

The effects of electro-elastic interactions on the phase velocities of quasi waves are observed for class 2 in Fig. 3. The phase velocity of $q P_{2}$ wave increases due to piezoelectric interaction while the effects are not significant in the case of other three waves.

(a)
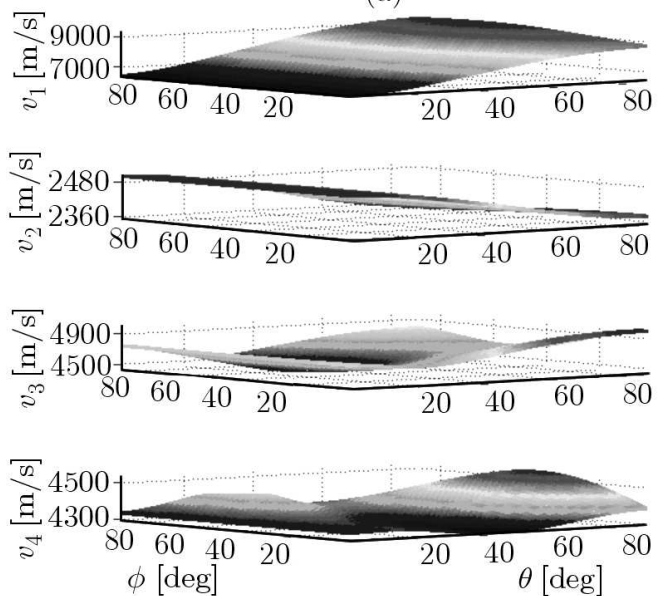

(b)
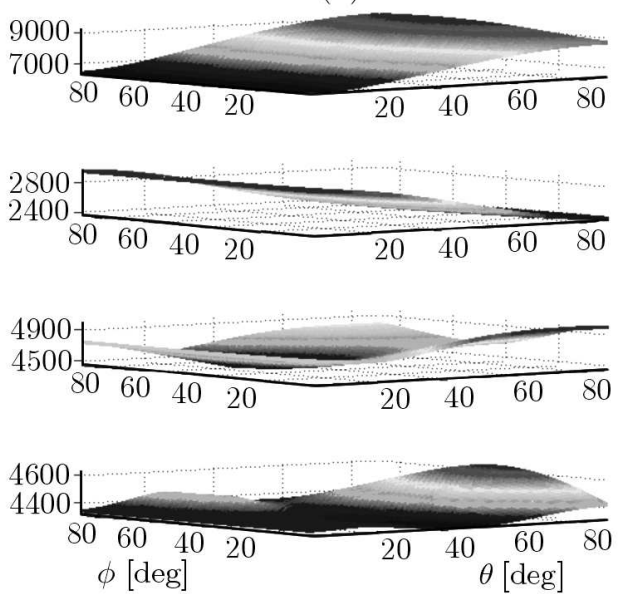

Fig. 3. Effects of piezoelectricity on the variation of phase velocities of quasi waves with the direction of propagation $(\theta, \phi)$ for Barium Sodium Niobate crystal of class 2; (a) without effects of piezoelectricity,

(b) with effects of piezoelectricity

Figure 4 shows the variation of phase velocities of $q P_{1}, q P_{2}, q S_{1}$ and $q S_{2}$ waves with the pore volume fraction in LFR and HFR, respectively, for class 2. It is observed that in the LFR, the phase velocities of all waves decrease with an increase in porosity while they increase monotonically by a very small amount with porosity in the HFR. The phase velocities of $q P_{1}$, $q S_{1}$ and $q S_{2}$ waves in the LFR become almost constant when the porosity is greater than $60 \%$. However, phase velocity of $q P_{2}$ wave decreases even when the porosity is greater than $60 \%$. Thus the slowest wave is found to be more sensitive to the porosity of the medium. 
(a)
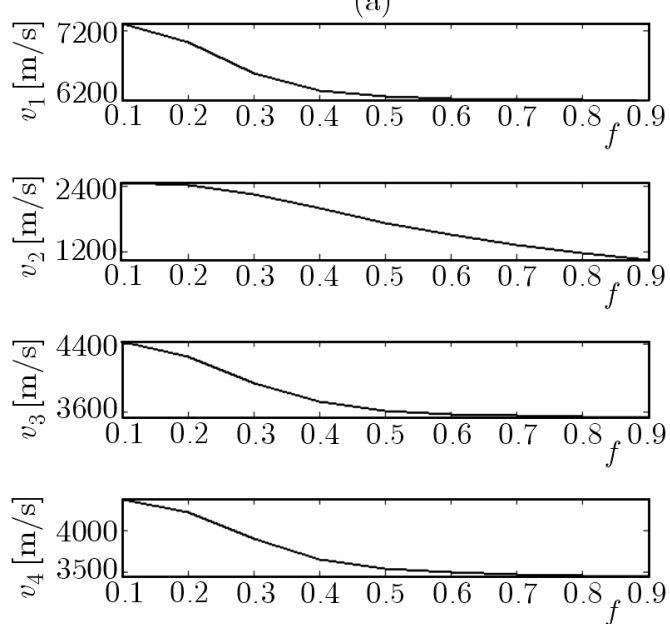

(b)
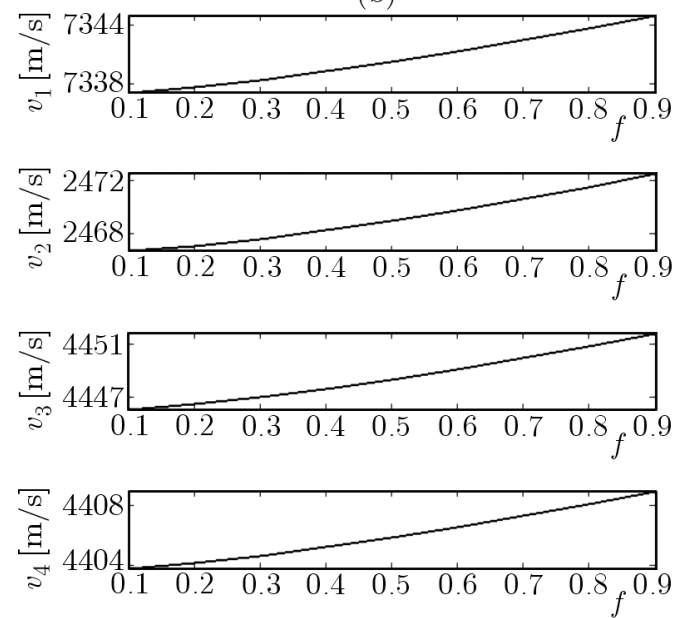

Fig. 4. Variation of phase velocities of quasi waves with porosity $f$ for Barium sodium Niobate crystal of class 2; (a) LFR, (b) HFR

\section{Conclusion}

In the present paper, wave propagation in a monoclinic porous piezoelectric material is studied both analytically and numerically. The four complex roots of the obtained Christoffel equation define the phase velocities of propagation of four stiffened quasi waves propagating in such a medium. The variation of phase velocities of these waves with frequency, phase direction and the porosity is observed numerically for a particular crystal Barium Sodium Niobate. The phase velocities of all four waves increase with frequency. The phase velocities of all four waves decrease with porosity in LFR which can be explained on the basis of percolation theory. The electric-elastic interaction does not affect the behavior of phase velocities with porosity but the magnitude of quasi P2 wave increases significantly.

\section{References}

1. Arnau A. (EDs.), 2008, Piezoelectric Transducers and Applications, Springer

2. Auld B.A., 1973, Acoustic Fields and Waves in Solids, Vol. 1, John Wiley \& Sons, Inc.

3. Auld B.A., 1981, Wave propagation and resonance in piezoelectric materials, Journal of Acoustic Society of America, 70, 6, 1577-1585

4. Craciun F., Guidarelli G., Galassi C., Roncari E., 1998, Elastic wave propagation in porous piezoelectric ceramics, Ultrasonic, 36, 427-430

5. Gomez T.E., Mulholland A.J., Hayward G., Gomatam J., 2000, Wave propagation in 03/3-3 connectivity composites with complex microstructure, Ultrasonics, 38, 897-907

6. Gupta R.K., Venkatesh T.A., 2006, Electromechanical response of porous piezoelectric materials, Journal Acta Materialia, 4063-4078

7. NAYfen A.H., Chien H.T., 1992, Wave propagation interaction with free and fluid loaded piezoelectric substrates, Journal of Acoustic Society of America, 91, 3126-3135

8. Piazza D., Stoleriu L., Mitoseriu L., Stancu A., Galassi C., 2006, Characterization of porous PZT ceramics by first order reversal curves (FORC) diagrams, Journal of the European Ceramic Society, 26, 2959-2962

9. Praveenkumar B., Kumar H.H., Kharat D.K., 2005, Characterization and microstructure of porous lead zirconate titanate ceramics, Bulletin Material Sciences, 28, 5, 453-455 
10. Qian Z., Jin F., Kishimoto K., Wang Z., 2004, Effect of initial stress on the propagation behavior of $\mathrm{SH}$ waves in multilayered piezoelectric composite structures, Sensor and Actuators A, 112, 368-375

11. Vashishth A.K., Gupta V., 2009a, Vibration of porous piezoelectric plates, Journal of Sound and Vibration, 325, 781-797

12. Vashishth A.K., Gupta V., 2009b, Wave propagation in transversely isotropic porous piezoelectric materials, International Journal of Solids and Structures, 46, 3620-3632

13. Zinchuk L.P., Podlipenets A.N., 2001, Dispersion equations for Rayleigh waves in a piezoelectric periodically layered structure, Journal of Mathematical Sciences, 103, 3, 398-403

Manuscript received February 10, 2015; accepted for print September 29, 2015 